\title{
Vertebral fractures are increased in rheumatoid arthritis despite recent therapeutic advances: a case-control study
}

\author{
N. Guañabens ${ }^{1}$ (I) · J. M. Olmos ${ }^{2}$ • J. L. Hernández ${ }^{2} \cdot$ D. Cerdà ${ }^{3} \cdot$ C. Hidalgo Calleja ${ }^{4}$ • J. A. Martinez López ${ }^{5}$. \\ L. Arboleya ${ }^{6} \cdot$ F. J. Aguilar del Rey ${ }^{7}$ S. Martinez Pardo ${ }^{8} \cdot$ I. Ros Vilamajó ${ }^{9} \cdot$ X. Suris Armangué ${ }^{10} \cdot$ D. Grados ${ }^{11}$. \\ C. Beltrán Audera ${ }^{12} \cdot$ E. Suero-Rosario ${ }^{13} \cdot$ I. Gómez Gracia ${ }^{14}$ - A. Salmoral Chamizo ${ }^{14} \cdot$ I. Martín-Esteve ${ }^{13} \cdot$ H. Florez ${ }^{1}$. \\ A. Naranjo ${ }^{15}$. S. Castañeda ${ }^{16}$. S. Ojeda Bruno ${ }^{15} \cdot$ S. García Carazo ${ }^{17}$. A. García Vadillo ${ }^{16} \cdot$ L. López Vives ${ }^{18}$. \\ À. Martínez-Ferrer ${ }^{19} \cdot$ H. Borrell Paños ${ }^{18} \cdot$ P. Aguado Acín ${ }^{17} \cdot$ R. Castellanos-Moreira ${ }^{1} \cdot$ C. Tebé $^{20}$. \\ C. Gómez-Vaquero ${ }^{21}$ - for the OsteoResSer Working Group of the Spanish Society of Rheumatology
}

Received: 23 September 2020 / Accepted: 4 January 2021 / Published online: 18 January 2021

(C) International Osteoporosis Foundation and National Osteoporosis Foundation 2021

\begin{abstract}
Summary Prevalence and risk factors of vertebral fractures in postmenopausal RA women were assessed in 323 patients and compared with 660 age-matched women. Of patients, 24.15\% had at least one vertebral fracture vs.16.06\% of controls. Age, glucocorticoids and falls were the main fracture risks. Vertebral fractures were associated with disease severity.

Introduction There is little quality data on the updated prevalence of fractures in rheumatoid arthritis (RA) that may have changed due to advances in the therapeutic strategy in recent years. This study was aimed at analysing the prevalence and risk factors of vertebral fractures in postmenopausal women with RA and comparing it with that of the general population.

Methods We included 323 postmenopausal women diagnosed with RA from 19 Spanish Rheumatology Departments, randomly selected and recruited in 2018. Lateral radiographs of the thoracic and lumbar spine were obtained to evaluate morphometric vertebral fractures and the spinal deformity index. We analysed subject characteristics, factors related to RA, and fracture risk factors. The control group consisted of 660 age-matched Spanish postmenopausal women from the population-based Camargo cohort.

Results Seventy-eight (24.15\%) RA patients had at least one vertebral fracture. RA patients had increased fracture risk compared with controls $(106$ of $660,16.06 \%)(p=0.02)$. Logistic regression analysis showed that age $($ OR $2.17 ; 95 \%$ CI $1.27-4.00)$, glucocorticoids (OR 3.83; 95\% CI 1.32-14.09) and falls (OR 3.57; 95\% CI 1.91-6.86) were the independent predictors of vertebral fractures in RA patients. The subgroup with vertebral fractures had higher disease activity (DAS28: 3.15 vs. $2.78, p=$ 0.038 ) and disability (HAQ: 0.96 vs. $0.63, p=0.049$ ), as compared with those without vertebral fractures.

Conclusion The risk of vertebral fracture in RA is still high in recent years, when compared with the general population. The key determinants of fracture risk are age, glucocorticoids and falls. Patients with vertebral fractures have a more severe RA.
\end{abstract}

Keywords Osteoporosis $\cdot$ Rheumatoid arthritis $\cdot$ Vertebral fractures

\section{Key messages}

- The risk of vertebral fracture is currently higher in rheumatoid arthritis than in the general population.

- Independent variables for vertebral fractures in rheumatoid arthritis are age, treatment with glucocorticoids and falls.

- RA patients with vertebral fractures have higher disease activity and greater disability than those without fractures.

\section{N. Guañabens}

nguanabens@ub.edu

Extended author information available on the last page of the article

\section{Introduction}

A concept that has been transmitted for years is that rheumatoid arthritis (RA) is associated with a twofold increased risk of osteoporosis and fractures when compared with the general population $[1,2]$. This increased risk occurs in all age segments and in both sexes. Thus, more than a decade ago, Van Staa et al. found an increased risk of fracture in a cohort of 30,262 RA patients from the British General Practice Research Database. The risk was more marked at the spine and hip, with relative risk (RR) of 2.4 (95\% CI 2.0-2.8) and 
2.0 (95\% CI 1.8-2.3), respectively, when compared with control patients without a history of RA [3]. A recent meta-analysis, which included 13 studies published between 1993 and 2014, again showed a higher risk of fracture in patients with RA than in patients without RA (RR: 2.25 ; 95\% IC 1.762.87 , with a particularly high risk of vertebral and hip fractures [4].

There is little data on the prevalence of vertebral fractures in RA patients. Recently, two studies have analysed this aspect and the data are somewhat discordant. The prevalence described in a large cohort of Chinese RA patients was $20.2 \%$, but this study lacked a control group; thus, it is difficult to estimate whether this prevalence is higher than that of the general population [5]. Another study, which was unicentric, described a prevalence of $36.4 \%$ in a short series of German women, compared to $22.6 \%$ in the control population [6].

The introduction of a more aggressive therapeutic strategy for RA goes back two decades. Thus, the introduction of biologic DMARDs, potent inhibitors of the pro-inflammatory cytokines, revolutionised the treatment of this chronic rheumatic disease. Biologic DMARDs have a significant influence on the reduction of inflammation and bone resorption, as shown in several studies assessing the effects of TNF- $\alpha$ and IL-6 inhibitors [7] on bone remodelling. The effects of rituximab (anti-CD20) or abatacept (anti-CTLA4-Ig) on bone remodelling parameters have been scarcely analysed. Taking it all together, it seems logical that if inflammation decreases and bone resorption declines, bone mass should increase or be maintained. This is the case with several studies basically carried out with infliximab, but also with etanercept, adalimumab and tocilizumab [7-9]. However, other studies do not support a reduction in the prevalence of osteoporosis in the last 10 to 15 years, nor an effect of tumour necrosis factor (TNF)- $\alpha$ inhibitors in bone mineral density (BMD) and fractures [10-12]. Thus, Hauser et al. evaluated the prevalence and clinical predictors of osteoporosis in 304 RA patients between 2009 and 2010, reporting a prevalence of $29.9 \%$, compared with $17.4 \%$ in age-matched controls from the general population [10].

Risk factors for fracture and systemic bone loss have been extensively analysed in RA patients in studies performed over the years. In this sense, subject characteristics such as age and body mass index (BMI), spine and hip BMD and both disease activity and duration are the main markers of risk, depending on the studies $[3,10]$. Other medications in addition to biologic DMARDs may influence bone loss and fracture. Accordingly, it is well known that glucocorticoids are a major risk factor for fractures in the general population, as well as in patients with RA $[3,12,13]$.

The objective of this study was to analyse the prevalence of fractures in a group of patients with RA in a clinical practice setting and compare it with that of the general population and also, to analyse if in the last 5 years there has been a change in the factors that influence the risk of fracture, compared to those historically proposed.

\section{Methods}

\section{Subjects}

In this case-control study, we included 330 postmenopausal women diagnosed with RA, fulfilling the 2010 American College of Rheumatology/European League against Rheumatism classification criteria [14], from 19 Spanish Rheumatology Departments. Patients were randomly selected using computer randomisation from the registry of RA patients in each centre and were recruited between January and December 2018. Seven patients were excluded because radiographs of the spine were not available. The control group consisted of 660 age-matched Spanish postmenopausal women, in a 1:2 ratio, from the Camargo cohort, a communitybased population of more than 1,000 healthy postmenopausal women attending primary care centres in Northern Spain, included between 2006 and 2008 [15, 16].

All RA patients provided a written informed consent to participate, and the study was approved by the Ethics Committee of the University Hospital of Bellvitge, Catalonia, Spain.

\section{Procedures}

Lateral radiographs of the thoracic and lumbar spine were obtained from all patients at the time of the study or in the 6 prior months in order to evaluate morphometric vertebral fractures. Vertebral fractures were classified into mild (grade 1: reduction of height of 20-25\%), moderate (grade 2: reduction of 26-40\%) and severe (grade 3: reduction $>40 \%$ ), according to the Genant grading scale [17]. The spinal deformity index (SDI) was calculated by summing up the grade of each vertebra for every patient (from 0 to 3 ) from T4 to L4 [18].

Data on BMD at the lumbar spine, femoral neck and total hip measured by dual-energy X-ray absorptiometry (DXA) (Lunar or Hologic) were collected from the medical records of patients with RA and expressed as standardised BMD values and as T-scores. In the control group, BMD values using Hologic QDR 4500 (Bedford, MA, USA) were collected from all women included in the study. Individual vertebral bodies were excluded from the analysis if vertebral fracture was present.

The fracture assessment tool (FRAX) was calculated in 275 RA patients for estimating the individualised 10-year probability of major and hip fracture risk. 


\section{Risk factor assessment}

The study variables included subject characteristics, indicators of disease severity (in the RA group) and risk factors for fracture. Subject characteristics were age and body mass index (BMI). Factors related to RA were disease duration, erosive disease, rheumatoid factor (RF), anti-citrullinated peptide antibodies (ACPAs), C-reactive protein and both 28-joint Disease Activity Score (DAS28-ESR) and disability by the Health Assessment Questionnaire (HAQ) in the previous 5 years. Antirheumatic drugs included synthetic and biologic disease-modifying antirheumatic drugs (DMARDs). The assessed fracture risk factors were prior fragility fracture, parental history of hip fracture, smoking history, alcohol intake, glucocorticoid use, secondary osteoporosis and time since menopause. Prior treatment with any bone active agent was recorded. We recorded the number of falls in the 5 years previous to the radiological assessment of vertebral fractures. We used the data as a continuous variable (number of falls) and as a categorical variable (presence or absence of falls).

\section{Statistical analysis}

Baseline characteristics of postmenopausal women diagnosed with RA were described using mean and standard deviation or median and interquartile range for continuous variables and frequencies for categorical variables. Clinical characteristics in RA women were compared according to the presence of prevalent vertebral fractures using a $t$ test or Wilcoxon test for continuous variables and a chi-square test for categorical variables. The association of disease-related risk factors in RA patients with vertebral fractures was assessed using a logistic regression model. The association between the SDI and clinical fracture risk factors in RA patients was assessed estimating Pearson or Spearman correlation coefficient and testing the null hypothesis of rho $=0$. Finally, the presence of clinical fracture risk factors was compared between RA and control patients, using a $t$ test or Wilcoxon test for continuous variables and a chi-square test for categorical variables. The analysis was carried out using the statistical program $\mathrm{R}$, version 3.5.1.

\section{Results}

\section{Characteristics of study participants}

The median age of the patients was $69(61-75)$ years and the disease duration was 12.6 (7.3-20.6) years. As shown in Table 1, 79\% and $76 \%$ of patients had positive RF and ACPA, respectively. Erosive RA was diagnosed in $55 \%$ of patients and the median DAS28 and HAQ score in the previous 5 years was $2.8(2.37-3.42)$ and $0.75(0.12-1.40)$,
Table 1 Characteristics of RA patients

\begin{tabular}{lc}
\hline & $N=323$ \\
\hline Disease duration (years) & $12.6(7.3-20.6)$ \\
Rheumatoid factor + ve (\%) & $255(79)$ \\
ACPA + ve (\%) & $236(76)$ \\
DAS28-ESR ( $n=184)^{*}$ & $2.8(2.4-3.4)$ \\
HAQ (n=185)* & $0.75(0.12-1.40)$ \\
CRP (mg/L) & $4.8(2.5-8.4)$ \\
Erosive disease (\%) & $174(55)$ \\
Synthetic DMARDs & $313(96.9)$ \\
Methotrexate (\%)* & $249(75.5)$ \\
Leflunomide (\%) & $97(30)$ \\
Hydroxychloroquine $(\%)$ & $90(28)$ \\
Biologic DMARDs (\%) & $132(40.9)$ \\
Anti-TNFa (\%) & $98(30)$ \\
Tocilizumab (\%) & $34(11)$ \\
Abatacept (\%) & $29(9)$ \\
Rituximab (\%) & $19(6)$ \\
Glucocorticoids & \\
Daily prednisone dose (mg) & $2.2(0-5.0)$ \\
Cumulative prednisone dose (g) & $7.3(1.0-16.8)$ \\
\hline
\end{tabular}

*Previous 5 years. Data are expressed as median and interquartile range DAS28 28-joint Disease Activity Score, HAQ Health Assessment Questionnaire, $A C P A$ anti-cyclic citrullinated peptide antibodies, $C R P$ C-reactive protein, DMARD disease-modifying antirheumatic drug, $T N F$ tumour necrosis factor

respectively. Almost half (41.1\%) of the patients were in remission (DAS28<2.6), $28.1 \%$ had low disease activity (DAS28: $2.6-3.2$ ), $28.1 \%$ had moderate disease activity (DAS28: $3.2-5.1$ ) and only $2.7 \%$ of patients had a high disease activity (DAS28 > 5.1).

Two hundred and seventy-two (84.2\%) patients had received oral glucocorticoids at some stage of their disease and $71.4 \%$ in the 5 years prior to their inclusion in the study. Most patients had received treatment with at least one conventional synthetic DMARD, with methotrexate being the most commonly used (75.5\%), with a median duration of 60 (3060) months, in the5-year period of analysis. Of patients, $40.9 \%$ used a biological agent; $98(30 \%)$ of these patients had received anti-TNF $\alpha$ therapy. Nearly one-third (36\%) of patients had been previously treated with bisphosphonates, $14 \%$ with denosumab and 3\% with teriparatide. More than half of patients had received supplements of calcium and/or vitamin D.

\section{Vertebral fractures and disease-related risk factors in RA patients}

Seventy-eight (24.15\%) out of 323 patients with RA had at least one vertebral fracture. Patients with RA had an increased 
risk of fracture compared with age-matched controls (106 out of $660,16.06 \%)(p=0.02)$. The prevalence of a single vertebral fracture was similar in patients with RA and controls ( $11.15 \%$ vs $11.52 \%)$; however, patients with RA had more often two $(5.26 \%$ vs. $2.73 \%)$, three or more fractures than controls (7.74\% vs. $1.82 \%)$. The median value of SDI in RA patients was $3.0(1.75-5.0)$ and the most frequently fractured vertebrae were T12, L1 and L2, while in controls they were T6, T7 and L1. Patients who had been treated for osteoporosis most often had vertebral fractures. Accordingly, vertebral fractures were observed in $42(36.8 \%)$ out of 114 patients treated with bisphosphonates, $19(43.2 \%)$ out of 44 patients treated with denosumab and in the 8 patients treated with teriparatide.

Table 2 shows the differences between patients with RA, according to whether they had vertebral fractures. As observed, patients with vertebral fractures were older, and most often were over 65 . The postmenopausal period was longer, and they had a more frequent history of previous fractures (excluding vertebral fractures) and falls when compared with those without fractures. In addition, the subgroup of RA patients with vertebral fractures had higher disease activity and greater disability than those without vertebral fractures. Thus, patients with fractures had a median DAS28 of 3.15 and HAQ of 0.96 versus 2.78 and 0.63 , respectively. Patients with fractures had received glucocorticoids more frequently, and the cumulative dose was higher than that of patients without vertebral fractures.

The association of disease-related risk factors in RA patients with prevalent vertebral fractures is shown in Table 3. Vertebral fractures were associated with erosive disease, HAQ in the previous 5 years, use of glucocorticoids and treatment with rituximab. DAS28 was associated with vertebral fractures, although the association was not significant. There
Table 3 Association of disease-related risk factors in RA patients with vertebral fractures

\begin{tabular}{lcr}
\hline & OR $(95 \%$ CI $)$ & $\mathrm{p}$ \\
\hline Disease duration & $1.02(1.0-1.04)$ & 0.09 \\
Rheumatoid factor + ve & $0.92(0.5-1.69)$ & 0.79 \\
ACPA + ve & $0.97(0.3-1.78)$ & 0.92 \\
Erosive disease & $\mathbf{1 . 8 3}(\mathbf{1 . 1}-\mathbf{3 . 1 2})$ & $\mathbf{0 . 0 0 3}$ \\
DAS28-ESR* & $1.46(0.99-2.15)$ & 0.058 \\
HAQ* & $\mathbf{1 . 5 6}(\mathbf{1 . 0 - 2 . 4 4 )}$ & $<\mathbf{0 . 0 5}$ \\
Disease activity & & \\
Low activity & $1.07(0.4-2.88)$ & 0.89 \\
Moderate/high activity & $2.29(0.97-5.42)$ & 0.058 \\
Glucocorticoid treatment & $\mathbf{3 . 2 1}(\mathbf{1 . 2 2}-\mathbf{8 . 4})$ & $\mathbf{0 . 0 1 8}$ \\
Synthetic DMARs & & \\
Methotrexate & $1.63(0.69-3.84)$ & 0.26 \\
Leflunomide & $0.88(0.5-1.54)$ & 0.65 \\
Biologic DMARs & & \\
TNF- $\alpha$ inhibitors & $0.74(0.42-1.32)$ & 0.31 \\
Tocilizumab & $1.17(0.52-2.62)$ & 0.71 \\
Abatacept & $1.24(0.53-2.92)$ & 0.62 \\
Rituximab & $\mathbf{3 . 1}(\mathbf{1 . 2 2}-\mathbf{7 . 9 3})$ & $\mathbf{0 . 0 1 8}$ \\
\hline
\end{tabular}

*Previous 5 years

Low disease activity, DAS28 $\geq 2.6$ and $\leq 3.2$; moderate disease activity, DAS28 $>3.2$ and $\leq 5.1$; high disease activity, DAS28 $>5.1$ (ref. 52)

Statistical significance is shown in bold

ACPA anti-cyclic citrullinated peptide antibodies, DAS28 28-joint Disease Activity Score, $H A Q$ Health Assessment Questionnaire, $D M A R D$ disease-modifying antirheumatic drug, TNF tumour necrosis factor

was no association between prevalent vertebral fractures and disease duration, nor whether patients were in remission or

Table 2 Clinical characteristics according to the presence of vertebral fractures in RA patients

\begin{tabular}{|c|c|c|c|}
\hline & $\begin{array}{l}\text { Without VF } \\
(n=245)\end{array}$ & $\begin{array}{l}\text { With VF } \\
(n=78)\end{array}$ & $p$ \\
\hline Age (years) & $66(60-73)$ & $74(69-79)$ & $<0.001$ \\
\hline Age $\geq 65$ years & $108(44.1 \%)$ & $66(84.6 \%)$ & $<0.001$ \\
\hline DAS28-ESR $(n=69)^{*}$ & $2.78(2.35-3.26)$ & $3.15(2.39-3.75)$ & 0.032 \\
\hline $\operatorname{HAQ}(n=184)^{*}$ & $0.63(0.13-1.38)$ & $0.96(0.25-1.85)$ & 0.049 \\
\hline Glucocorticoid treatment & $193(78.8 \%)$ & $72(93.5 \%)$ & 0.008 \\
\hline Cumulative prednisone dose $(\mathrm{g})^{*}$ & $5.8(1.2-15.2)$ & $11.1(5.0-28.1)$ & 0.054 \\
\hline Previous fragility fracture (VF excluded) & $41(16.7 \%)$ & $32(41 \%)$ & $<0.001$ \\
\hline Postmenopausal period (years) & $17(11.0-25.0)$ & $27(21.0-31.0)$ & $<0.001$ \\
\hline Falls* & $77(31.4 \%)$ & $44(62.9 \%)$ & $<0.001$ \\
\hline
\end{tabular}

*Previous 5 years

Data are expressed as median and interquartile range

$V F$ vertebral fracture, DAS28 28-joint Disease Activity Score, HAQ Health Assessment Questionnaire 
with moderate or high disease activity. We did not find any association with positivity of RF or ACPA and their levels (scale). Correlations of the SDI with risk factors are summarised in Table 4.

When we classified patients according to the disease duration, no differences in the prevalence of vertebral fractures were observed in patients with more or less than 10 years of RA evolution ( $26 \%$ versus $21.3 \%, p=0.200$ ). In patients with less than 10 years of evolution, vertebral fractures were associated with DAS28 (OR 1.69; 95\% CI 1.03-2.77) and with moderate/severe disease activity (OR 3.78; 95\% CI 1.2511.49) as well as with the use of glucocorticoids. Like that which was found when analysing the whole patient group, rituximab was associated with vertebral fractures.

The multivariate logistic model for the prediction of vertebral fractures in patients with RA showed that the independent variables were age (OR 2.17; 95\% CI 1.27-4.00), the use of glucocorticoids (OR 3.83; 95\% CI 1.32-14.09) and previous falls (OR 3.57; 95\% CI 1.91-6.86). The same independent predictors of SDI were found.

\section{Classical risk factors for fracture in RA patients compared with controls}

Differences in classic risk factors for fracture were assessed in both RA patients and the control group (Table 5). No significant differences in the time from menopause was observed, nor in the family history of hip fracture between RA patients and the control group. However, there were significant differences in other risk factors, such as previous fracture and, as expected, being a current smoker and exposure to glucocorticoids, which were more common in the RA group. Patients with RA had significantly lower BMD and T-score values at the lumbar spine, femoral neck and total hip than the control group. Likewise, more patients with RA had osteoporosis, according to the WHO classification criteria [19] than controls

Table 4 Correlations of the spinal deformity index with risk factors in RA patients

\begin{tabular}{lcc}
\hline & $r$ & $p$ \\
\hline Age (years) & 0.267 & $<0.001$ \\
Mean HAQ & 0.223 & $<0.001$ \\
Cumulative GC dose (g)* & 0.115 & $<0.05$ \\
Daily GC dose (mg)* & 0.115 & $<0.05$ \\
Femoral neck BMD (g/cm2) & -0.146 & $<0.05$ \\
Total hip BMD (g/cm2) & -0.135 & $<0.05$ \\
Postmenopausal period (years) & 0.286 & $<0.001$ \\
Number of falls* & 0.354 & $<0.001$ \\
\hline
\end{tabular}

*Previous 5 years

$H A Q$ Health Assessment Questionnaire, $G C$ glucocorticoids, $B M D$ bone mineral density
( $37.96 \%$ vs. $23.64 \%$ ), with the value of BMD being classified as normal in $11.31 \%$ and $20.61 \%$, respectively.

Age, particularly above 65 years, was associated with vertebral fractures in both groups (ORs 2.22; 95\% CI 1.66-2.96 and $1.87 ; 95 \%$ CI $1.50-2.33, p<0.001)$ as well as the time from menopause. Previous fragility fracture, treatment with glucocorticoids and being a current smoker were significantly associated risk factors only in the group with RA (ORs ranging from 0.5 to 7.01). As was predictable, vertebral fractures were significantly associated with osteoporosis (OR 3.65; 95\% CI 1.78-7.48, $p<0.001)$ and BMD at the lumbar spine, femoral neck and total hip in the Camargo cohort. However, no association was found between vertebral fractures and osteoporosis according to DXA criteria in patients with RA. Only BMD and T-score at the femoral neck were associated with vertebral fractures in the RA population $(p=0.03)$.

\section{Discussion}

The results of this study show that in the last few years, the prevalence of vertebral fractures in postmenopausal women with RA is higher than that of women of the same age in the general population. Furthermore, the number of fractures in patients with RA is higher than in the control group. This result may be surprising, considering that the therapeutic strategy in RA in the last decades has significantly modified longterm disease outcomes. In this respect, numerous studies have shown that synthetic and particularly biologic DMARDs may stop bone loss associated with systemic inflammation [20]. However, not all authors have found this beneficial effect [10]. Recent data referring to the prevalence of fractures are scarce, particularly those referring to vertebral fractures, as most series are focused on non-vertebral fractures [21-23]. Notably, Ozen et al. in 2019 showed a reduction in the risk of vertebral fracture in RA patients treated with anti-TNF $\alpha$ [13]. A Swedish study found an increased risk of fragility fractures in RA in both the 1990s and 2000s, despite patients in the 2000s receiving potent pharmacological treatment early in the disease. This study, however, was not focused on vertebral fractures, but on other fragility fractures such as upper arm, hip and other fractures [22]. Similarly, an upward trend in the incidence of hip fracture in Spanish RA patients between 1999 and 2015 was reported, despite a decreasing age-adjusted incidence in the Spanish general population $[24,25]$.

When analysing the risk factors for fracture in RA, it is common that the risk increases with age in most studies, including the present study, in which age was an independent predictor of fracture. Moreover, patients with RA and vertebral fractures were older than patients without fractures ( $74 \mathrm{vs}$. 66 years). Another association to highlight in our series were falls. Although falls can be related to advanced age [26], it has 
Table 5 Demographics and risk factors for fracture in patients with RA and controls

\begin{tabular}{|c|c|c|c|}
\hline & Rheumatoid arthritis & Controls & \\
\hline & $(N=323)$ & $(N=660)$ & $p$ \\
\hline Age (years) & $69(61-75)$ & $69(62-78)$ & n.s. \\
\hline$>65$ years $(\%)$ & $203(62.8)$ & $428(64.9)$ & n.s. \\
\hline BMI $\left(\mathrm{kg} / \mathrm{m}^{2}\right)$ & $26.2(23.5-29.6)$ & $28.1(25.4-31.3)$ & $<0.001$ \\
\hline Years from menopause & $14(7-22)$ & $13(6-22)$ & n.s. \\
\hline Family history of hip fracture (\%) & $43(13.3)$ & $68(10.3)$ & n.s. \\
\hline Previous fracture (\%) & $95(29.4)$ & $109(16.5)$ & $<0.001$ \\
\hline Current smoker (\%) & $102(31.6)$ & $60(9.1)$ & $<0.001$ \\
\hline Alcohol intake $(\%)$ & $8(2.5)$ & $75(11.4)$ & $<0.001$ \\
\hline Glucocorticoid treatment (\%) & $272(84.2)$ & $15(2.3)$ & $<0.001$ \\
\hline \multicolumn{4}{|l|}{ Bone mineral density } \\
\hline Lumbar BMD $\left(\mathrm{g} / \mathrm{cm}^{2}\right)$ & $0.923(0.82-1.038)$ & $0.971(0.882-1.068)$ & $<0.001$ \\
\hline Lumbar T-score & $-1.90(-2.73$ to -0.94$)$ & $-1.62(-2.36$ to -0.77$)$ & 0.009 \\
\hline Femoral neck BMD $\left(\mathrm{g} / \mathrm{cm}^{2}\right)$ & $0.730(0.662-0.816)$ & $0.786(0.711-0.878)$ & $<0.001$ \\
\hline Femoral neck T-Score & $-1.70(-2.22$ to -1.05$)$ & $-1.27(-1.84$ to -0.57$)$ & $<0.001$ \\
\hline Total hip BMD $\left(\mathrm{g} / \mathrm{cm}^{2}\right)$ & $0.798(0.711-0.883)$ & $0.858(0.774-0.939)$ & $<0.001$ \\
\hline Total hip T-score & $1.28(-1.09$ to -0.59$)$ & $-0.79(-1.48$ to -0.13$)$ & $<0.001$ \\
\hline FRAX-MOF $(n=275)$ & $9.1(4.45-18.0)$ & & \\
\hline FRAX-Hip $(n=275)$ & $2.3(0.8-7.1)$ & & \\
\hline
\end{tabular}

Data are expressed as median and interquartile range

$B M I$ body mass index, $B M D$ bone mineral density, $M O F$ major osteoporotic fracture

been historically reported that patients with RA fall more frequently than non-RA individuals [27]. A recent study performed in UK population confirmed that, even in the last decade, RA has been associated with a self-reported increase in number of falls in the previous year, which was up to $23.1 \%$ [28].

A major objective of our study was to analyse whether vertebral fractures were associated with duration and a greater activity of the disease. In this sense, the duration of the disease was not associated with the presence of fractures in the overall RA group, nor when we divided patients according to whether the disease was longstanding or had less than 10 years of evolution. In contrast, van Staa et al. found an increased risk of fracture in patients with longstanding RA ( $>10$ years' duration), although in their series this increased risk was more marked at the hip [3].

Our findings are consistent with a substantially elevated risk of fracture in patients with more active disease, and particularly in those with worse physical function. Although the independent predictors of vertebral fractures did not include both clinical measures, DAS28 and HAQ were higher in RA patients with vertebral fractures than in those without, and we observed a significant association of vertebral fractures with erosive disease and the HAQ score. DAS28 was also associated with vertebral fracture, although this did not reach statistical significance. This relationship between vertebral fracture and disease activity should be taken with caution, as we found that vertebral fracture was not associated with low, moderate or high disease activity. We expected to find an increased fracture risk in patients with more active disease and a higher degree of systemic inflammation. Indeed, Vis et al. found a relationship between clinical response, in terms of reduced inflammatory activity, and changes in bone loss at the spine, particularly at the hip. In this series however, the risk of fracture was not assessed [29]. Perhaps the cross-sectional nature of our study did not allow this aspect to be properly grasped. Another possible explanation is that the number of patients with moderate and particularly high disease activity was low. Thus, almost $70 \%$ of patients were in remission or had low disease activity and only $2.7 \%$ had high disease activity.

There is good clinical evidence that ACPA-positive RA patients develop more bone erosions than those who are ACPA-negative. In addition, there is also some evidence that bone strength is decreased, and systemic bone loss is more severe in ACPA-positive RA patients [30]. The concomitant presence of high levels of RF further enhances the risk of bone loss in patients with early disease [31]. In our study, we did not find an association of vertebral fractures with positivity of RF or ACPAs and their levels, nor was the presence of ACPA or RF more common in patients with vertebral fractures than in non-fractured patients. Similarly, other authors have not found an association between seropositivity for RF or ACPAs and osteoporosis in RA patients either [10]. 
The pharmacological treatment of RA has two cornerstones: glucocorticoids and DMARDs. The results of this study confirm the harmful effects of glucocorticoids on bone in RA $[32,33]$. Although this does not seem innovative, it has its own significance, as it has been speculated whether glucocorticoids, by inhibiting inflammation, could bring benefits in the context of localised and generalised bone loss as well as in disability, when used in the active phase of the disease [34-38]. Our results reinforce that at least at the generalised skeletal level, glucocorticoids are independent predictors of vertebral fracture and their use is more common in patients with vertebral fractures than in those without. Indeed, in our series, like in others [7, 20], BMD was lower in RA patients than in controls. The effect of glucocorticoids on fracture development could explain, at least in part, the finding that BMD values at the lumbar spine and total hip were not associated with fracture in the RA group. By contrast, in the control group, BMD values at the lumbar spine, femoral neck and total hip were significantly associated with vertebral fractures. In this sense, it is known that in glucocorticoid-induced osteoporosis, fractures develop with BMD values higher than those of postmenopausal women, frequently in patients with nonosteoporotic T-score values. This suggests that bone quality rather than quantity could be decisive in the risk of fracture in this context [39-43]. Furthermore, a recent study found that $26.3 \%$ of RA patients with vertebral fractures had a normal BMD in the lumbar spine and that a high proportion of women in their series had low values of trabecular bone score (TBS) [6]. Another study, conducted in South Korea, showed that there was a significant difference in TBS values between RA patients with vertebral fractures compared to those without fractures. In this series, no significant difference in BMD was observed between patients with and without fractures and TBS values were negatively correlated with the cumulative dose of glucocorticoids, but not with RA disease activity parameters [44]. Interestingly, TBS has been shown to have greater discriminative power than BMD for fracture risk assessment in glucocorticoid-treated patients [45].

Another aspect to highlight in this study is that treatment with most synthetic or biological DMARDs was not associated with fracture development. This was expected, as most but not all studies show that the use of DMARDs is associated with a decrease in bone loss, with preservation or increase in spine and hip BMD and a better profile in bone turnover markers [20, 46, 47]. This has been particularly demonstrated with anti-TNF blocking agents, infliximab being the most analysed biologic. Indeed, antiTNF agents and tocilizumab may have a favourable effect on local bone balance allowing for some bone formation, since both agents have limited potential in inducing the repair of bone erosions in RA patients [48, 49].
Only three studies conducted up to 2015 had analysed the effect on fractures after TNF inhibitor treatment. The reported data showed no difference in the risk of nonvertebral fractures in RA patients treated with TNF inhibitors, methotrexate or other non-biologic DMARDs, although one study reported a decreased fracture risk with anti-TNF monotherapy [50]. However, only isolated studies analysed the impact of DMARDs on vertebral fractures. In our study, we found that the use of rituximab was associated with the development of vertebral fractures. It has been suggested that the B lymphocyte depletion could protect RA patients from bone loss, since B cells are a major source of RANKL. Wheater et al. analysed the effect of rituximab on BMD and bone turnover markers in a small group of RA patients over a 12month period. There was a significant decrease in femoral neck and total hip BMD with preservation of BMD at the lumbar spine and no significant change in the resorption marker. The authors suggested different hypotheses to explain these data, highlighting the influence of vitamin D status and perhaps the requirement for prednisolone as a confusing factor [51].

The strengths of this study include a comparator design involving age-matched women from the same country at a ratio of $1: 2$, the same radiological criteria of vertebral fracture for patients and controls and the multicentric nature of our cohort from specialised rheumatology centres. Limitations include the fact that due to the retrospective nature of this study and the strict criterion of assessing the median DAS28 and HAQ scores in the previous 5 years, we were not able to obtain these data from all our patients. Other limitations of the study include that a nonnegligible percentage of patients had received antiosteoporosis drugs at some point in their evolution. This data, however, does not undervalue the higher prevalence of vertebral fractures found in our group of RA patients when compared to the control population, although it may have influenced BMD values. Finally, it would have been of value to have TBS measurements and serum 25hydroxyvitamin D levels in our population.

In conclusion, this study shows that the prevalence of vertebral fractures is higher in women with RA compared to the general age-matched population. The main message of our results is that despite advances in the management of RA in recent decades, particularly after the introduction of biologic drugs, the increased risk of vertebral fracture persists. Being older, receiving glucocorticoids and having falls in the previous 5 years are the independent predictors of vertebral fracture in our series of RA patients. We cannot, however, rule out that a greater degree of disability, radiological damage and increased disease activity contribute to the risk of vertebral fracture. 
Funding This study is funded in part by a grant from the Instituto de Salud Carlos III (PI18/00762), Ministerio de Economia y Competitividad, Spain, that included FEDER funds from the EU.

\section{Compliance with ethical standards}

Conflicts of interest Dr. Guañabens has in the past received fees for lectures and/or advice from Alexion, Amgen, Eli Lilly and UCB. Dr. Olmos has received in the past fees for lectures from Amgen, Eli Lilly, Stada and Gedeon-Richter. Dr. Hernandez received grant funding and fees for lectures from AMGEN and MSD. Dr. Naranjo has in the past received fees for lectures and/or advice from Amgen and UCB. Dr. Castañeda has received fees for lectures and/or advice from Amgen, Eli Lilly, MSD, Novartis, Roche, Stada and UCB. Dr. Castellanos-Moreira has in the past received fees for lectures and/or advice from Pfizer, Lilly, $\mathrm{UCB}$ and BMS. The remaining authors have no competing interests to disclose.

Ethical approval The study was approved by the Ethics Committee of the University Hospital of Bellvitge, Catalonia, Spain.

Informed consent All RA patients provided a written informed consent to participate.

\section{References}

1. Amin S, Gabriel SE, Achenbach SJ, Atkinson EJ, Melton LJ 3rd. (2013) Are young women and men with rheumatoid arthritis at risk for fragility fractures? A population-based study. J Rheumatol 40(10): 1669-1676

2. Kim SY, Schneeweiss S, Liu J, Daniel GW, Chang CL, Garneau K et al (2010) Risk of osteoporotic fracture in a large populationbased cohort of patients with rheumatoid arthritis. Arthritis Res Ther 12(4):R154

3. van Staa TP, Geusens P, Bijlsma JW, Leufkens HG, Cooper C (2006) Clinical assessment of the long-term risk of fracture in patients with rheumatoid arthritis. Arthritis Rheum 54(10):3104-3112

4. Xue AL, Wu SY, Jiang L, Feng AM, Guo HF, Zhao P (2017) Bone fracture risk in patients with rheumatoid arthritis: a meta-analysis. Medicine (Baltimore) 96(36):e6983

5. Tong JJ, Xu SQ, Zong HX, Pan MJ, Teng YZ, Xu JH (2020) Prevalence and risk factors associated with vertebral osteoporotic fractures in patients with rheumatoid arthritis [published correction appears in Clin Rheumatol. 2020 Jun;39(6):2023]. Clin Rheumatol 39(2):357-364

6. Buehring B, Thomas J, Wittkämper T, Baraliakos X, Braun J (2020) Evaluation of the trabecular bone score (TBS) in routine clinical care of patients with inflammatory rheumatic and noninflammatory diseases: correlation with conventional bone mineral density measurement and prevalence of vertebral fractures. Z Rheumatol. https://doi.org/10.1007/s00393-020-00764-9

7. Dubrovsky AM, Lim MJ, Lane NE (2018) Osteoporosis in rheumatic diseases: anti-rheumatic drugs and the skeleton. Calcif Tissue Int 102(5):607-618

8. Ricciardi BF, Paul J, Kim A, Russell LA, Lane JM (2013) Osteoporosis drug therapy strategies in the setting of diseasemodifying agents for autoimmune disease. Osteoporos Int 24(2): 423-432

9. Briot K, Rouanet S, Schaeverbeke T, Etchepare F, Gaudin P, Perdriger A et al (2015) The effect of tocilizumab on bone mineral density, serum levels of Dickkopf-1 and bone remodeling markers in patients with rheumatoid arthritis. Joint Bone Spine 82(2):109 115

10. Hauser B, Riches PL, Wilson JF, Horne AE, Ralston SH (2014) Prevalence and clinical prediction of osteoporosis in a contemporary cohort of patients with rheumatoid arthritis. Rheumatology (Oxford) 53(10):1759-1766

11. Siu S, Haraoui B, Bissonnette R, Bessette L, Roubille C, Richer V et al (2015) Meta-analysis of tumor necrosis factor inhibitors and glucocorticoids on bone density in rheumatoid arthritis and ankylosing spondylitis trials. Arthritis Care Res (Hoboken). 67(6):754 764

12. Kawai VK, Grijalva CG, Arbogast PG, Curtis JR, Solomon DH, Delzell E et al (2013) Initiation of tumor necrosis factor $\alpha$ antagonists and risk of fractures in patients with selected rheumatic and autoimmune diseases. Arthritis Care Res (Hoboken) 65(7):10851094

13. Ozen G, Pedro S, Wolfe F, Michaud K (2019) Medications associated with fracture risk in patients with rheumatoid arthritis. Ann Rheum Dis 78(8):1041-1047

14. Aletaha D, Neogi T, Silman AJ, Funovits J, Felson DT, Bingham CO 3rd et al (2010) 2010 Rheumatoid arthritis classification criteria: an American College of Rheumatology/European League Against Rheumatism collaborative initiative [published correction appears in Ann Rheum Dis. 2010 Oct;69(10):1892]. Ann Rheum Dis 69(9): $1580-1588$

15. Martínez J, Olmos JM, Hernández JL, Pinedo G, Llorca J, Obregón E et al (2009) Bone turnover markers in Spanish postmenopausal women: the Camargo cohort study. Clin Chim Acta 409(1-2):70 74

16. Olmos JM, Hernández JL, García-Velasco P, Martínez J, Llorca J, González-Macías J (2016) Serum 25-hydroxyvitamin D, parathyroid hormone, calcium intake, and bone mineral density in Spanish adults. Osteoporos Int 27(1):105-113

17. Genant HK, Wu CY, van Kuijk C, Nevitt MC (1993) Vertebral fracture assessment using a semiquantitative technique. J Bone Miner Res 8(9):1137-1148

18. Kerkeni S, Kolta S, Fechtenbaum J, Roux C (2009) Spinal deformity index (SDI) is a good predictor of incident vertebral fractures. Osteoporos Int 20(9):1547-1552

19. Kanis JA (1994) Assessment of fracture risk and its application to screening for postmenopausal osteoporosis: synopsis of a WHO report. WHO Study Group. Osteoporos Int 4(6):368-381

20. Zerbini CAF, Clark P, Mendez-Sanchez L, Pereira RMR, Messina OD, Uña CR et al (2017) Biologic therapies and bone loss in rheumatoid arthritis. Osteoporos Int 28(2):429-446

21. Lin $\mathrm{YC}, \mathrm{Li} \mathrm{YH}$, Chang $\mathrm{CH}, \mathrm{Hu} \mathrm{CC}$, Chen DW, Hsieh $\mathrm{PH}$ et al (2015) Rheumatoid arthritis patients with hip fracture: a nationwide study. Osteoporos Int 26(2):811-817. https://doi.org/10.1007/ s00198-014-2968-y

22. Nyhäll-Wåhlin BM, Ajeganova S, Petersson IF, Andersson M (2019) Increased risk of osteoporotic fractures in Swedish patients with rheumatoid arthritis despite early treatment with potent disease-modifying anti-rheumatic drugs: a prospective general population-matched cohort study. Scand J Rheumatol 48(6):431438

23. Mazzucchelli R, Pérez Fernandez E, Crespí-Villarías N, QuirósDonate J, García Vadillo A, Espinosa M et al (2018) Trends in hip fracture in patients with rheumatoid arthritis: results from the Spanish National Inpatient Registry over a 17-year period (19992015). TREND-AR study. RMD Open 4(1):e000671

24. Mazzucchelli R, Pérez Fernández E, Crespí Villarías N, Tejedor Alonso MÁ, Sáez López P et al (2019) East-west gradient in hip fracture incidence in Spain: how much can we explain by following the pattern of risk factors? Arch Osteoporos 14(1):115 
25. Pueyo-Sánchez MJ, Larrosa M, Suris X, Casado E, Auleda J, Fusté $\mathrm{J}$ et al (2017) Secular trend in the incidence of hip fracture in Catalonia, Spain, 2003-2014. Age Ageing 46(2):324-328

26. Soukola SK, Jämsen ERK, Pauniaho SLK, Ukkonen MT (2020) A population-based study of 2347 fall-related injuries among older people in a Finnish emergency department. Eur Geriatr Med 11(2):315-320

27. Armstrong C, Swarbrick CM, Pye SR, O'Neill TW (2005) Occurrence and risk factors for falls in rheumatoid arthritis. Ann Rheum Dis 64(11):1602-1604

28. Clynes MA, Jameson K, Prieto-Alhambra D, Harvey NC, Cooper C, Dennison EM (2019) Impact of rheumatoid arthritis and its management on falls, fracture and bone mineral density in UK Biobank. Front Endocrinol (Lausanne) 10:817

29. Vis M, Havaardsholm EA, Haugeberg G, Uhlig T, Voskuyl AE, van de Stadt RJ et al (2006) Evaluation of bone mineral density, bone metabolism, osteoprotegerin and receptor activator of the NFkappaB ligand serum levels during treatment with infliximab in patients with rheumatoid arthritis. Ann Rheum Dis 65(11): 1495-1499

30. Stemmler F, Simon D, Liphardt AM, Englbrecht M, Rech J, Hueber AJ et al (2018) Biomechanical properties of bone are impaired in patients with ACPA-positive rheumatoid arthritis and associated with the occurrence of fractures. Ann Rheum Dis 77(7):973-980

31. Bugatti S, Bogliolo L, Vitolo B, Manzo A, Montecucco C, Caporali R (2016) Anti-citrullinated protein antibodies and high levels of rheumatoid factor are associated with systemic bone loss in patients with early untreated rheumatoid arthritis. Arthritis Res Ther 18(1): 226

32. Kim D, Cho SK, Park B, Jang EJ, Bae SC, Sung YK (2018) Glucocorticoids are associated with an increased risk for vertebral fracture in patients with rheumatoid arthritis. J Rheumatol 45(5): $612-620$

33. Wang Y, Zhao R, Gu Z, Dong C, Guo G, Li L (2020) Effects of glucocorticoids on osteoporosis in rheumatoid arthritis: a systematic review and meta-analysis. Osteoporos Int 31(8):1401-1409

34. Ibañez M, Ortiz AM, Castrejón I, Garcia-Vadillo JA, Carvajal I, Castañeda $\mathrm{S}$ et al (2010) A rational use of glucocorticoids in patients with early arthritis has a minimal impact on bone mass. Arthritis Res Ther 12(2):R50

35. Sambrook PN, Cohen ML, Eisman JA, Pocock NA, Champion GD, Yeates MG (1989) Effects of low dose corticosteroids on bone mass in rheumatoid arthritis: a longitudinal study. Ann Rheum Dis 48(7):535-538

36. Habib GS, Haj S (2005) Bone mineral density in patients with early rheumatoid arthritis treated with corticosteroids. Clin Rheumatol 24(2):129-133

37. Güler-Yüksel M, Hoes JN, Bultink IEM, Lems WF (2018) Glucocorticoids, inflammation and bone. Calcif Tissue Int 102(5): 592-606

38. Blavnsfeldt AG, de Thurah A, Thomsen MD, Tarp S, Langdahl B, Hauge EM (2018) The effect of glucocorticoids on bone mineral density in patients with rheumatoid arthritis: a systematic review and meta-analysis of randomized, controlled trials. Bone. 114:172180
39. Peel NF, Moore DJ, Barrington NA, Bax DE, Eastell R (1995) Risk of vertebral fracture and relationship to bone mineral density in steroid treated rheumatoid arthritis. Ann Rheum Dis 54(10):801806

40. Luengo M, Picado C, Del Rio L, Guañabens N, Montserrat JM, Setoain J (1991) Vertebral fractures in steroid dependent asthma and involutional osteoporosis: a comparative study. Thorax 46(11):803-806

41. van Staa TP, Laan R, Barton IP, Cohen V, Reid DM, Cooper C (2003) Bone density threshold and other predictors of vertebral fracture in patients receiving oral glucocorticoid therapy. Arthritis Rheum 48(11):3224-3229

42. Kanis JA, Johansson H, Oden A, Johnell O, de Laet C, Melton C III et al (2004) A meta-analysis of prior corticosteroid use and fracture risk. J Bone Miner Res 19(6):893-899

43. Compston J (2018) Glucocorticoid-induced osteoporosis: an update. Endocrine. 61(1):7-16

44. Choi YJ, Chung YS, Suh CH, Jung JY, Kim HA (2017) Trabecular bone score as a supplementary tool for the discrimination of osteoporotic fractures in postmenopausal women with rheumatoid arthritis. Medicine (Baltimore) 96(45):e8661

45. Florez H, Hernández-Rodríguez J, Muxi A, Carrasco JL, PrietoGonzález S, Cid MC et al (2020) Trabecular bone score improves fracture risk assessment in glucocorticoid-induced osteoporosis. Rheumatology (Oxford) 59(7):1574-1580

46. Confavreux CB, Chapurlat RD (2011) Systemic bone effects of biologic therapies in rheumatoid arthritis and ankylosing spondylitis. Osteoporos Int 22(4):1023-1036

47. Raterman HG, Lems WF (2019) Pharmacological management of osteoporosis in rheumatoid arthritis patients: a review of the literature and practical guide. Drugs Aging 36(12):1061-1072

48. Finzel S, Rech J, Schmidt S, Engelke K, Englbrecht M, Stach C et al (2011) Repair of bone erosions in rheumatoid arthritis treated with tumour necrosis factor inhibitors is based on bone apposition at the base of the erosion. Ann Rheum Dis 70(9):1587-1593

49. Finzel S, Rech J, Schmidt S, Engelke K, Englbrecht M, Schett G (2013) Interleukin-6 receptor blockade induces limited repair of bone erosions in rheumatoid arthritis: a micro CT study. Ann Rheum Dis 72(3):396-400

50. Coulson KA, Reed G, Gilliam BE, Kremer JM, Pepmueller PH (2009) Factors influencing fracture risk, T score, and management of osteoporosis in patients with rheumatoid arthritis in the Consortium of Rheumatology Researchers of North America (CORRONA) registry. J Clin Rheumatol 15(4):155-160

51. Wheater G, Elshahaly M, Naraghi K, Tuck SP, Datta HK, van Laar JM (2018) Changes in bone density and bone turnover in patients with rheumatoid arthritis treated with rituximab, results from an exploratory, prospective study. PLoS One 13(8):e0201527

52. Anderson J, Caplan L, Yazdany J, Robbins ML, Neogi T, Michaud $\mathrm{K}$ et al (2012) Rheumatoid arthritis disease activity measures: American College of Rheumatology recommendations for use in clinical practice. Arthritis Care Res (Hoboken). 64(5):640-647

Publisher's note Springer Nature remains neutral with regard to jurisdictional claims in published maps and institutional affiliations. 


\section{Affiliations}

N. Guañabens ${ }^{1}$ (I) - J. M. Olmos ${ }^{2} \cdot$ J. L. Hernández ${ }^{2} \cdot$ D. Cerdà ${ }^{3} \cdot$ C. Hidalgo Calleja ${ }^{4} \cdot$ J. A. Martinez López ${ }^{5}$.

L. Arboleya ${ }^{6} \cdot$ F. J. Aguilar del Rey ${ }^{7} \cdot$ S. Martinez Pardo $^{8} \cdot$ I. Ros Vilamajó ${ }^{9} \cdot X$. Suris Armangué ${ }^{10} \cdot$ D. Grados $^{11}$.

C. Beltrán Audera ${ }^{12} \cdot$ E. Suero-Rosario ${ }^{13} \cdot$ I. Gómez Gracia ${ }^{14}$ - A. Salmoral Chamizo ${ }^{14} \cdot$ I. Martín-Esteve ${ }^{13} \cdot$ H. Florez ${ }^{1}$.

A. Naranjo ${ }^{15} \cdot$ S. Castañeda ${ }^{16}$. S. Ojeda Bruno ${ }^{15} \cdot$ S. García Carazo ${ }^{17}$. A. García Vadillo ${ }^{16} \cdot$ L. López Vives ${ }^{18}$.

À. Martínez-Ferrer ${ }^{19} \cdot$ H. Borrell Paños ${ }^{18} \cdot$ P. Aguado Acín ${ }^{17} \cdot$ R. Castellanos-Moreira ${ }^{1} \cdot$ C. Tebé $^{20}$.

C. Gómez-Vaquero ${ }^{21}$ • for the OsteoResSer Working Group of the Spanish Society of Rheumatology

1 Department of Rheumatology, Hospital Clínic, CIBERehd, IDIBAPS, University of Barcelona, Barcelona, Spain

2 Departament of Internal Medicine, University Hospital Marqués de Valdecilla-IDIVAL, Universidad de Cantabria, Santander, Spain

3 Department of Rheumatology, Hospital Moisès Broggi, Sant Joan Despí, University of Barcelona, Barcelona, Spain

4 Department of Rheumatology, University Hospital de Salamanca, Salamanca, Spain

5 Department of Rheumatology, University Hospital Fundación Jiménez Díaz, Madrid, Spain

6 Department of Rheumatology, University Hospital Central de Asturias, Oviedo, Spain

7 Department of Rheumatology, University Hospital Virgen de la Victoria, Malaga, Spain

8 Department of Rheumatology, University Hospital Mutua Terrassa, Barcelona, Spain

9 Department of Rheumatology, Hospital Son Llàtzer, Palma de Mallorca, Spain

10 Department of Rheumatology, Hospital General de Granollers, Granollers, International University of Catalunya, Barcelona, Spain

11 Department of Rheumatology, Hospital d'Igualada, Igualada, Barcelona, Spain
12 Department of Rheumatology, University Hospital Miguel Servet, Zaragoza, Spain

13 Department of Rheumatology, Hospital General Mateu Orfila, Maó, Spain

14 Department of Rheumatology, University Hospital Reina Sofía, Cordoba, Spain

15 Department of Rheumatology, University Hospital de Gran Canaria Dr. Negrín, Las Palmas de Gran Canaria, Spain

16 Department of Rheumatology, University Hospital La Princesa IISIP, Madrid, Spain

17 Department of Rheumatology, University Hospital La Paz, Madrid, Spain

18 Department of Rheumatology, Hospital Sant Rafael, Barcelona, Spain

19 Department of Rheumatology, University Hospital Doctor Peset, Valencia, Spain

20 Biostatistics Unit, Bellvitge Biomedical Research Institute (IDIBELL) L'Hospitalet de Llobregat, Barcelona, Spain

21 Department of Rheumatology, University Hospital de Bellvitge, L'Hospitalet de Llobregat, Barcelona, Spain 